\title{
Опыт диагностики и лечения внутрипочечных опухолей
}

\author{
С. Б. Имамвердиев, Э. Д. Гасымов, Э. Н. Эфендиев \\ Азербайджанский медицинский университет, г. Баку
}

\section{Experience of diagnosis and treatment of intrarenal tumors}

\author{
S. B. Imamverdiyev, E. D. Gasimov, E. N. Afandiyev \\ Azerbaijani Medical University, Baku
}

\begin{abstract}
Реферат
Цель. Выбор оптимального метода хирургического лечения внутрипочечных опухолей и расширение показаний к органосохраняющим операциям.

Материалы и методы. В исследование, проведенное на клинических базах кафедры урологии Азербайджанского медицинского университета - Республиканской клинической больницы имени академика Мирасадуллы Миркасимова и Центральной больницы моряков, включили 37 пациентов с внутрипочечными опухолями, которых лечили в этих клиниках в 2006 - 2016 гг. Мужчин было 17 (45,9\%), женщин - 20 (54,1\%).

Результаты. У 11 (29,7\%) пациентов диагностировали опухоль верхнего полюса почки, у 19 (51,3\%) - нижнего, у 7 $(18,9 \%)$ - среднего сегмента почки. У 26 (70,1\%) пациентов установили внепочечный тип опухоли, у 11 (29,9\%) - внутрипочечный с прорастанием в более глубокие слои паренхимы почки. Размеры опухолей у 24 пациентов варьировали от 2,5 до 4,0 см, у 13 - от 4,0 до 7,0 см. Резекцию почки выполнили у 20 (54,1\%) пациентов (у 13 - справа, у 7 - слева), энуклеацию опухоли - у 10 (27\%) пациентов (у 3 - справа, у 7 - слева), энуклеорезекцию - у 7 (18,9\%) пациентов (у 4 - справа, у 3 - слева)

Выводы. Органосохраняющие операции выполнили у всех 37 пациентов с внутрипочечными опухолями стадии Т1 Т2. Успех выполненных операций зависел от своевременной диагностики, точной оценки риска, правильного выбора типа органосохраняющих вмешательств и профессионализма хирурга.

Ключевые слова: внутрипочечная опухоль; органосохраняющие операции.

Abstract

Objective. The choice of the surgical treatment method for intrarenal tumors and enhancing of indications for organ-preserving operations.

Materials and methods. In the investigation, conducted on the Urology Cathedra clinical bases of Azerbaijani Medical University - Republican Clinical Hospital named after Academician Mirasadulla Mirkasimov and Central Hospital for Sailors, were included 37 patients with intrarenal tumors, who were treated in these clinics in $2006-2016$ yrs. There were 17 (45.9\%) men and $20(54.1 \%)$ women.

Results. In 11 (29.7\%) patients the tumor of upper renal pole was diagnosed, in $19(51.3 \%)$ - of the lower one, in 7 (18.9\%) of the middle renal segment. In 26 (70.1\%) patients the intrarenal tumoral type was established, in 11 (29.9\%) - the intrarenal one with penetration in deeper layers of renal parenchyma. The tumors dimensions in 24 patients have varied from 2.5 to 4.0 $\mathrm{cm}$, in 13 - from 4.0 to $7.0 \mathrm{~cm}$. Renal resection was performed in 20 (54.1\%) patients (in 13 - a right-sided, in 7 - left-sided), enucleation of tumor - in 10 (27\%) patients (in 3 - a right-sided, in 7 - a left-sided), enucleoresection - in 7 (18.9\%) patients (in 4 - a right-sided, in 3 - a left-sided).

Conclusion. Organ-preserving operations were performed in all 37 patients with intrarenal tumors Stages T1 - T2. Success of the operations performed have depended upon timely diagnosis, precise estimation of risk, correct choice of the organ-preserving operation type and a surgeon qualification.

Keywords: intrarenal tumor; organ-preserving operations.
\end{abstract}

Рак почки длительное время протекает бессимптомно, поэтому так важны его ранняя диагностика, определение распространенности процесса, что позволяет выбрать оптимальный метод оперативного лечения [1, 2]. Появление новых современных и совершенствование уже имеющихся методов лучевой диагностики, применение их в клинической практике как по отдельности, так и при необходимости в комплексе позволили в 60-70\% больных определять ранние стадии рака почки, а развитие хирургической науки и технологий привело к появлению новых видов оперативных вмешательств и изменило отношение клиницистов к радикальной нефрэктомии, которую на протяжении многих лет считали «золотым стандартом» лечения рака почки [3]. Врачи стали рассматривать органосохраняющие операции как более предпочтительные. Органосохраняющие операции при раке почек выполняют примерно 20\% больным [3 5]. Возможность выполнения органосохраняющей операции при раке почки прежде всего зависит от локализации и размера опухоли. По данным исследования, проведенного Американским национальным медицинским институтом, органосохраняющая операция возможна на почке, если размер опухоли не превышает 3 см. Однако есть данные о выполнении этих операций и при опухолях размером, достигающим 4 см [6]. Органосохраняющие операции на почках выполняют как открыто, так и лапароскопически. Одни авторы считают возможным лапароскопическое удаление опухоли почки размером 
до 3 см при условии ее внепочечного роста, другие - до 5 см, причем даже при некоторых формах внутрипочечного роста опухоли $[7,8]$.

Для сохранения органа более важным параметром, чем размер опухоли, являются особенности ее расположения в почке и направление роста $[9,10]$. Большинство специалистов считают целесообразным и возможным сохранять орган при внепочечном направлении роста опухоли почки. Выполнение подобных операций при внутрипочечном росте опухоли остается предметом больших дискуссий среди клиницистов.

Качество выполненной органосохраняющей операции при раке почки определяется частотой возникновения локальных рецидивов, а также продолжительностью жизни больных. Рецидив после органосохраняющих операций развивается у 5,6 - 18\% больных [10, 11]. Большинство авторов рекомендует проводить линию резекции почки на расстоянии 1 см от края опухоли, так как при этом частота возникновения локальных рецидивов самая низкая 5,6\%. По мнению некоторых исследователей, вероятность появления локальных рецидивов рака почки достигает 10\% вследствие нерадикальности выполненной резекции. Появление местных рецидивов рассматривают также как недостаток органосохраняющих операций. Часть исследователей видит причину местного метастазирования в больших размерах опухоли.

Видами открытых органосохраняющих операций при раке почки в зависимости от размера и особенностей роста опухоли могут быть: резекция почки, энуклеация и энуклеорезекция опухоли [5, 7]. Если раньше органосохраняющие операции выполняли только у больных с поражением опухолями обеих почек, с единственной или с единственно функционирующей почкой, то в последние десятилетия показания к органосохраняющим операциям при опухолях почек значительно расширили.

Цель исследования: выбор оптимального метода хирургического лечения внутрипочечных опухолей и расширение показаний к органосохраняющим операциям.

\section{Материалы и методы исследования}

В исследование, проведенное на клинических базах кафедры урологии Азербайджанского медицинского университета - Республиканской клинической больницы имени академика Мирасадуллы Миркасимова и Центральной больницы моряков, включили 37 больных, которым в этих клиниках в 2006 - 2016 гг. были выполнены органосохраняющие операции по поводу рака почек. Мужчин было 17 (45,9\%), женщин - 20 (54,1\%).

\section{Результаты}

Опухоли почек стадии Т1N0M0 наблюдали у 10 (27,0\%) больных, T1aNOMO - у 6 (16,2\%), T1bNOMO - у 2 (5,4\%), T2NOMO - y $19(51,3 \%)$.

У 11 больных опухоль локализовалась в верхнем полюсе почки, у 19 - в нижнем, у 7 - в среднем сегменте почки. У 26 (70,3\%) больных наблюдали внепочечный, у 11 (29,7\%) - внутрипочечный тип роста опухоли без прорастания в глубину паренхимы почки. Размеры опухоли у 24 больных варьировали от 2,5 до 4,0 см, у 13 больных
- от 4,0 до 7,0 см. Резекцию почки выполнили 20 (54,1\%) больным (13 - справа, 7 - слева), энуклеацию опухоли 10 (27,0\%) больным (3 - справа, 7 - слева), энуклеорезекцию опухоли - 7 (18,9\%) (4 - справа, 3 - слева).

По данным патогистологического исследования установлены следующие диагнозы: почечно-клеточный рак у 12 (32,4\%) больных (G1 - у 4, G2 - у 2, G3 - у 3, GX - у 3); светло-клеточный рак - у 11 (29,7\%) больных (G1 - у 5, G2 - у 2, G3 - у 2, GX - у 2); аденокарцинома - у 7 (18,9\%) больных (G1 - у 2, G2 - у 2, G3 - у 2, GX - у 1); базофильноклеточный рак - у 3 (8,1\%) больных (G1 - у 2, GX - y 1); ангиомиолипосаркома - у 4 (10,8\%) больных (G1 - у 2, G2 - y 1, G3 - y 1).

Для улучшения результатов органосохраняющих операций мы следовали определенным принципам их выполнения. Это соблюдение правил абластичности выполнения операции, максимальное сбережение здоровой паренхимы почки, минимальное повреждение сосудов во избежание кровотечения и опытность оперирующего хирурга. Строгое соблюдение этих принципов при выполнении органосохраняющих операций у всех больных обеспечило отсутствие каких-либо серьезных осложнений в послеоперационном периоде и вполне удовлетворительное качество жизни.

\section{Обсуждение}

Точную локализацию опухоли в почке, внутриорганность или внеорганность роста, отношение к чашечнолоханочной системе у наблюдаемых нами больных достоверно определяли уже на начальном этапе диагностики с помощью ультразвукового исследования (УЗИ). Определение степени инвазии опухоли и направление ее роста являются ключевыми в дооперационной диагностике для принятия решения о характере органосохраняющей операции.

Мы в своей клинической практике у пациентов, у которых на основании проведенных лучевых методов диагностики диагностировали неинвазированные в окружающие ткани опухоли стадии Т1 - Т2, размещенные в полюсах или среднем сегменте почки с преимущественно внепочечным ростом, отдавали предпочтение органосохраняющим операциям. При внутрипочечном росте опухоли, поражающей лишь сегмент почки, и наличии сопутствующей патологии контрлатеральной почки также отдавали предпочтение сохранению органа. Приводим клиническое наблюдение.

Больной А., 43 лет, 21.10.2013 г. обратился в клинику с жалобами на тупую боль в поясничной области справа, общую слабость, повышение артериального давления до 21,3/12,0 кПа (160/90 мм рт. ст.). Считает себя больным около года. Лечения не получал. Женат, имеет двух детей.

Объективно: общее состояние удовлетворительное, на вопросы отвечает адекватно. Почки не пальпируются, симптом поколачивания справа слабо положительный. Общий анализ крови: гемоглобин 108 г/л, СОЭ 21 мм/ч. Биохимический анализ крови без изменений. Общий анализ мочи: лейкоциты покрывают все поле зрения. ЭКГ: синусовая брадикардия, гипертрофия левого желудочка, незначительные изменения передней стенки миокарда. 
УЗИ: печень и селезенка без изменений; размеры левой почки 8,6 × 4,1 см, толщина паренхимы 1,5 cм, чашечнолоханочная система расширена, в среднем сегменте имеется киста размерами 2,1 × 2,0 см; размеры правой почки 12,5 × 5,4 см, толщина паренхимы 1,6 cм, в верхнем и среднем сегментах имеется гипоэхогенное образование, не выходящее за контуры почки, размерами 5,5 × 4,3 см, чашечно-лоханочная система не расширена.

Для определения анатомо-функционального состояния почек больному проведена обзорная и экскреторная урография. На обзорной урограмме тени, подозрительные на конкременты, и объемные образования не определяются. На серии снимков экскреторной урографии функция обеих почек удовлетворительная, определяется симптом ампутации чашечек верхнего полюса правой почки, чашечно-лоханочная система правой почки незначительно расширена.

На основании проведенного обследования установлен диагноз: опухоль верхнего и среднего сегментов правой почки (T1NOMO); гипоплазия и киста левой почки.

23.10.2013 г. под эндотрахеальным наркозом больному выполнена операция - резекция верхнего полюса правой почки. Размер удаленной опухоли 5,3 × 4,4 см. Для уменьшения интраоперационной кровопотери резекция почки выполнена на фоне пережатия почечной артерии в течение 15 мин.

Осложнений в раннем послеоперационном периоде не наблюдали. Патогистологический диагноз: светлоклеточная аденокарцинома почки (pT1NOM0). Через 1,5 мес после операции для проверки функции почек больному проведена экскреторная урография: функция оперированной почки нормальная.

\section{Выводы}

Выполнение органосохраняющих операций оказалось возможным у всех 37 больных с опухолями почек стадии T1 - Т2. Успех выполненных операций зависел от своевременной диагностики, точной оценки риска, правильно выбранного вида органосохраняющего вмешательства и профессионализма хирурга.

\section{References}

1. Thompson RH, Lane BR, Lohse CM, Leibovich BC, Fergany A, Frank $\mathrm{I}$, et al. Every minute counts when the renal hilum is clamped during partial nephrectomy. Eur Urol. 2010 Sep;58(3):340-5. doi: 10.1016/j. eururo.2010.05.047.

2. Matveev VB, Volkova MI. Kidney cancer. RMZh. 2007;15(14):10949. [In Russian].

3. Say EV, Matveev VB, Volkova MI. Results of treatment of local recurrences of renal cell carcinoma. Onkourologiya. 2008;(1):9-15. [In Russian].

4. Pettus JA, Jang TL, Thompson RH, Yossepowitch O, Kagiwada M, Russo P. Effect of baseline glomerular filtration rate on survival in patients undergoing partial or radical nephrectomy for renal cortical tumors. Mayo Clin Proc. 2008 Oct;83(10):1101-6. doi: 10.4065/83.10.1101

5. Pereverzev AS, Shukin DV, Shcherbak AYu. Organ-preserving operations in renal cell carcinoma. Onkourologiya. 2009;(2):22-30. [In Russian].

6. Clark MA, Shikanov S, Raman JD, Smith B, Kaag M, Russo P, et al Chronic kidney disease before and after partial nephrectomy. J Urol. 2011;185(1):43-8. doi: 10.1016/j.juro.2010.09.019

7. Kleinmann N, Nadu A, Mor Y, Ramon G. Partial nephrectomy for centrally located tumor. Eur Urol Suppl 2006;5(2):181. Available from: https://www.eusupplements.europeanurology.com/article/S15699056(06)60642-8/pdf.

8. Gill IS, Patil MB, Abreu AL, Ng C, Cai J, Berger A, et al. Zero ischemia anatomical partial nephrectomy: a novel approach. J Urol. 2012 Mar;187(3):807-14. doi: 10.1016/j.juro.2011.10.146.

9. Carini M, Minervini A, Serni S. Nephron sparing surgery: current developments and controversies. Eur Urol. 2006;49:324-31. doi: 10.1016/j. eururo.2006.09.003.

10. Guzzo TJ, Pierorazio PM, Schaeffer EM, Fishman EK, Allaf ME. The accuracy of multidetector computerized tomography for evaluating tumor thrombus in patients with renal cell carcinoma. J Urol. 2009 Feb;181(2):486-90; discussion 491. doi: 10.1016/j.juro.2008.10.040.

11. Sobin LH. TNM Classification of maliginant tumors.UICC International Union Against Cancer. 7th edn. Wiley-Blackwell1.2009; 255-73. 